\title{
Utility of Early MRI in the Diagnosis and Management of Acute Spontaneous Intracerebral Hemorrhage
}

\author{
Christine A.C. Wijman ${ }^{a, b} \quad$ Chitra Venkatasubramanian ${ }^{a, b}$ Sara Bruins ${ }^{b}$ \\ Nancy Fischbein ${ }^{c}$ Neil Schwartz ${ }^{a}$ b \\ ${ }^{a}$ Department of Neurology and Neurological Sciences and ${ }^{\mathrm{b}}$ the Stanford Stroke Center, and ${ }^{\mathrm{c}}$ Department of \\ Radiology, Stanford University, Palo Alto, Calif., USA
}

\section{Key Words}

Cerebral hemorrhage $\cdot$ Magnetic resonance imaging •

Diagnostic imaging

\begin{abstract}
Background: The optimal diagnostic evaluation for spontaneous intracerebral hemorrhage (ICH) remains controversial. In this retrospective study, we assessed the utility of early magnetic resonance imaging (MRI) in $\mathrm{ICH}$ diagnosis and management. Methods: Eighty-nine (72\%) of 123 patients with spontaneous $\mathrm{ICH}$ underwent a brain $\mathrm{CT}$ and MRI within 30 days of $\mathrm{ICH}$ onset. Seventy patients with a mean age of 62 \pm 15 years were included. A stroke neurologist and a general neurologist, each blinded to the final diagnosis, independently reviewed the admission data and the initial head $\mathrm{CT}$ and then assigned a presumed ICH cause under 1 of 9 categories. ICH cause was potentially modified after subsequent MRI review. The final 'gold standard' ICH etiology was determined after review of the complete medical record by an independent investigator. Change in diagnostic category and confidence and the potential impact on patient management were systematically recorded. Results: Mean time to $\mathrm{MRI}$ was $3 \pm 5$ days. Final ICH diagnosis was hypertension or cerebral amyloid angiopathy (CAA) in $50 \%$ of patients. After MRI review the stroke neurologist changed diagnostic
\end{abstract}

category in $14 \%$, diagnostic confidence in an additional $23 \%$ and management in $20 \%$, and the general neurologist did so in 19, 21 and $21 \%$ of patients, respectively. MRI yield was highest in $\mathrm{ICH}$ secondary to ischemic stroke, CAA, vascular malformations and neoplasms, and did not differ by age, history of hypertension, hematoma location or the presence of intraventricular hemorrhage. Conclusions: The results of this study suggest potential additive clinical benefit of early MRI in patients with spontaneous ICH.

Copyright $\odot 2010$ S. Karger AG, Basel

\section{Introduction}

Spontaneous (i.e. nontraumatic) intracerebral hemorrhage (ICH) accounts for at least $10-20 \%$ of strokes worldwide [1]. Common etiologies are long-standing hypertension and cerebral amyloid angiopathy (CAA) $[2,3]$, but there are multiple other causes including vascular malformations [4], aneurysms, tumors, coagulopathy, cerebral venous thrombosis, hemorrhagic transformation of an ischemic infarct, illicit drug use [5], endocarditis, moyamoya disease [6] and vasculitis, among others.

Identification of the cause of ICH used to be based on clinical evaluation supported by brain computed tomography $(\mathrm{CT})$ with or without conventional catheter angi-

\section{KARGER}

(c) 2010 S. Karger AG, Basel

Fax +41613061234 E-Mail karger@karger.ch www.karger.com www.karger.com/ced
Christine A.C. Wijman, MD, $\mathrm{PhD}$

Department of Neurology and Neurological Sciences and the Stanford Stroke Center 701 Welch Road, B325

Palo Alto, CA 94034 (USA)

Tel. +1 650723 4448, Fax +1 650723 4451, E-Mail cwijman@ stanford.edu 
ography (CA) [7, 8]. It has been shown in recent years that magnetic resonance imaging (MRI) is just as sensitive as CT in the detection of acute brain hemorrhage [9-12] and more sensitive than $\mathrm{CT}$ in the detection of structural abnormalities [13-17]. While MRI has substantially improved our diagnostic capabilities, there are hardly any studies that have systematically evaluated its clinical effectiveness in ICH patients.

In this retrospective study we aimed to assess the clinical utility of early MRI in the diagnosis and management of ICH in a consecutive series of patients over a 5-year period at a single institution. The hypothesis was that MRI would be superior to CT in the diagnostic evaluation of patients presenting with a spontaneous $\mathrm{ICH}$, and that this additional information would translate into a change in patient management.

\section{Methods}

The Stanford Stroke Center database was reviewed for all patients with the diagnosis of ICH between January 1999 and February 2004. Patients were included if they had a diagnosis of spontaneous intraparenchymal or isolated intraventricular hemorrhage (IVH) and if they had undergone a brain CT and MRI within 30 days of symptom onset at our institution. The study protocol was approved by the Stanford University Medical Human Subjects Panel.

Brain MRIs were obtained on a 1.5-tesla GE Signa Horizon $\mathrm{NV} / \mathrm{i}$ scanner (EXCITE III) equipped with cardiac-enhanced gradients $(40 \mathrm{mT} / \mathrm{m})$. The following sequences were routinely obtained: $\mathrm{T}_{1^{-}}$(sagittal) and $\mathrm{T}_{2}$-weighted imaging (axial), $\mathrm{T}_{2}{ }^{*}$-weighted gradient echo MRI, diffusion-weighted imaging (DWI), fluidattenuated inversion recovery and 3-dimensional time-of-flight magnetic resonance angiography of the circle of Willis. $\mathrm{T}_{1-}$ weighted imaging with contrast and 3-dimensional time-of-flight magnetic resonance venography were obtained in some patients.

Admission and inpatient medical records were reviewed by one investigator, and the following information was documented on a standardized data form: age, sex, ethnicity, succinct history of present illness, history of hypertension, stroke or transient ischemic attack, atrial fibrillation, clotting disorders, renal failure, alcohol abuse, malignancy, diabetes mellitus, illicit drug use, smoking, home medications, pertinent family history including stroke, vascular malformations and bleeding disorders, and admission physical and neurological examination. Laboratory results including complete blood cell count, coagulation profile, comprehensive chemistry panel, urine toxicity screen and admission EKG results were also recorded. Patients were considered to have a history of hypertension when 1 of the following 3 criteria was met: well-documented history of hypertension, treatment with antihypertensive agents as an outpatient, and left ventricular hypertrophy by EKG or echocardiography. Coagulopathy was defined as a platelet count of $<50,000 \mathrm{cell} / \mathrm{s} / \mathrm{ml}$ or an international normalized ratio of $>3$ or an activated partial thromboplastin time of $>80 \mathrm{~s}$.

Early MRI in Acute Spontaneous

Intracerebral Hemorrhage
A stroke and a general neurologist - both blinded to the patients' clinical diagnoses and each other's readings - independently reviewed each patient's data form and the admission brain CT (including the neuroradiologist's report) and recorded a presumed ICH cause under 1 of 9 predefined categories. In addition they recorded their diagnostic confidence as 'possible', 'likely' or 'highly probable'. A potentially modified diagnosis or modified level of confidence was recorded after subsequent review of the brain MRI (which included the review of the neuroradiologist's report). If either the diagnosis or the level of confidence changed after review of the MRI, the evaluator was asked to record which MRI sequences were most relevant for the change (up to 3 were allowed) and if and how the change in ICH etiology or confidence would affect patient management.

The study neurologists were instructed to consider only MRI findings that have been validated to be highly specific for the diagnosis in question based on pathological verification in previous studies. These included for example cerebral microbleeds in the deep gray nuclei or brainstem suggestive of long-standing hypertension [15] or cerebral microbleeds in the subcortical white matter in an elderly patient with a lobar ICH suggestive of CAA [3, 16]. Hemorrhagic transformation of an ischemic stroke was considered if areas of restricted diffusion substantially exceeded the commonly seen perihemorrhagic diffusion artifacts or if there was presence of additional areas of restricted diffusion in other vascular territories in a patient with a compelling reason to suffer ischemic strokes (e.g. atrial fibrillation).

The final presumed ICH diagnosis or 'the gold standard diagnosis' was recorded by an independent investigator who was blinded to the diagnoses generated by the two study neurologists. This final diagnosis was determined after review of all available clinical, pathological and radiological data including clinic or imaging follow-up and typically corresponded to the clinical impression of the treating stroke neurologist(s) who cared for the patient. The CT and the MRI diagnoses of the study investigators were compared with this 'gold standard' diagnosis to determine whether the MRI added accuracy or certainty to the diagnostic impression. Credit for increased confidence was given only if diagnostic category did not change.

\section{Statistical Analysis}

Data analyses were performed using the SPSS 17.0 software package (SPSS, Chicago, Ill., USA). $\chi^{2}$ and Fisher's exact tests were used to analyze cross-categorized frequency data. All statistical tests were two-tailed, and statistical significance was defined at $\alpha<0.05$.

\section{Results}

During the study period, 89 (72\%) of 123 patients treated by the stroke service with a diagnosis of ICH had undergone both brain CT and MRI at our institution. Of these, 70 patients with a mean age of $62 \pm 15$ years are included in this report. Reasons for study exclusion were: the patient had a known ICH cause prior to admission (e.g. known untreated vascular malformation; $\mathrm{n}=17$ ) or 
Table 1. Demographics and hematoma location in 70 patients presenting with spontaneous ICH

\begin{tabular}{lc}
\hline Demographics & Number \\
\hline Male & $42(60)$ \\
Racial distribution & \\
American Indian or Alaska Native & $19(27)$ \\
Asian & $4(6)$ \\
Black or African American & $1(1)$ \\
Native Hawaiian or other Pacific Islander & $37(53)$ \\
White, not Hispanic or Latino & $9(13)$ \\
White, Hispanic or Latino & \\
ICH risk factors & $49(70)$ \\
Hypertension & $7(10)$ \\
Previous stroke or TIA & $9(13)$ \\
Alcohol use & $3(4)$ \\
Illicit drug use & $3(4)$ \\
Warfarin use & $11(16)$ \\
Aspirin use & \\
Hematoma location & $23(33)$ \\
Lobar & $17(24)$ \\
Basal ganglia & $9(13)$ \\
Thalamus & $2(3)$ \\
Brainstem & $7(10)$ \\
Cerebellum & $5(7)$ \\
Isolated IVH & $7(10)$ \\
Multiple hematomas & \\
\hline
\end{tabular}

Results are patient numbers, with percentages in parentheses. TIA $=$ Transient ischemic attack. the MRI was performed more than 30 days after the initial presentation $(n=2)$.

Patient characteristics are shown in table 1 . The mean time between symptom onset and the MRI was $3 \pm 5.4$ days. Of the 70 patients, 46 had contrast-enhanced $\mathrm{T}_{1^{-}}$ weighted imaging, and 6 patients had magnetic resonance venography in addition to the routine sequences listed above. Seventy-nine percent of hematomas were supratentorial. Twenty-four patients (34\%) had IVH; of these, 5 had isolated IVH. Seven patients had multiple hematomas, and 9 had associated subarachnoid hemorrhage shown by CT.

The final ICH cause was classified into 1 of 9 predefined categories based on the 'gold standard' as described above (table 2). Half of the patients had ICH attributed to either long-standing hypertension (36\%) or CAA (14\%). The MRI also helped identify other etiologies of spontaneous ICH such as vasculitis or hyperperfusion syndrome in 9 (13\%) patients. The ICH cause was considered unknown in $11 \%$ of patients despite extensive diagnostic workup, which included additional contrast CT, CT angiography and CA in most of these patients.

Twelve patients (17\%) had surgical evacuation of their hematomas, and a definite pathological diagnosis was obtained in 9 (brain tumor in 3 and vascular malformation in 6 patients). Eight of the 9 patients had been diagnosed correctly by preoperative MRI, including 1 patient with

Table 2. MRI impact on diagnostic category, diagnostic confidence and management in 70 patients with spontaneous ICH (average of 2 observers)

\begin{tabular}{lccc}
\hline Etiology of spontaneous ICH & $\begin{array}{l}\text { MRI changed } \\
\text { diagnostic } \\
\text { category } \\
(\mathrm{n}=11.5)\end{array}$ & $\begin{array}{l}\text { MRI changed } \\
\text { diagnostic } \\
\text { confidence } \\
(\mathrm{n}=15.5)\end{array}$ & $\begin{array}{l}\text { MRI changed } \\
\text { management } \\
(\mathrm{n}=14.5)\end{array}$ \\
\hline Hypertension $(\mathrm{n}=25)$ & $1(4 \%)$ & $5.5(22 \%)$ & $2(20 \%)$ \\
CAA $(\mathrm{n}=10)$ & $2.5(25 \%)$ & $2(29 \%)$ & $4(40 \%)$ \\
Vascular malformation $(\mathrm{n}=7)$ & $2(29 \%)$ & $0.5(17 \%)$ & $2(67 \%)$ \\
Neoplasm $(\mathrm{n}=3)$ & $2(67 \%)$ & $1.5(50 \%)$ & $2(67 \%)$ \\
Hemorrhagic transformation of an ischemic stroke $(\mathrm{n}=3)$ & $0.5(17 \%)$ & $0.5(17 \%)$ & 0 \\
Illicit drug use $(\mathrm{n}=3)$ & $0.5(25 \%)$ & $0.5(25 \%)$ & $0.5(25 \%)$ \\
Coagulopathy $(\mathrm{n}=2)$ & $1(13 \%)$ & $3(33 \%)$ & $0.5(6 \%)$ \\
Other identified cause $(\mathrm{n}=9)$ & $16 \%$ & 0 & $0.5(6 \%)$ \\
Unknown cause $(\mathrm{n}=8)$ & & $22 \%$ & $21 \%$ \\
\hline Total $(\mathrm{n}=70)$ & & & $20 \%$ \\
\hline
\end{tabular}



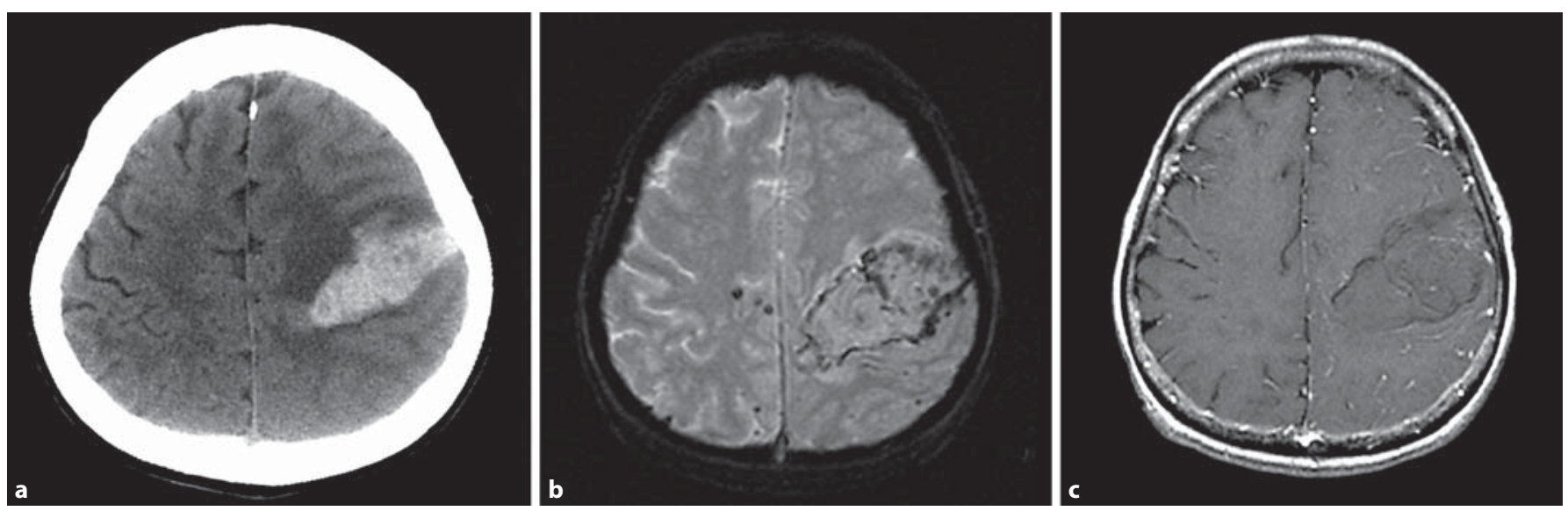

Fig. 1. Brain imaging studies in a 69-year-old hypertensive female with a spontaneous left frontoparietal lobar ICH. The CT diagnosis of ICH from a 'tumor' or 'unknown etiology' (a) was changed to ICH from CAA after the MRI review due to the presence of peripheral microbleeds on gradient echo MRI (b) and lack of enhancement on the $\mathrm{T}_{1}$-weighted postcontrast MRI (c).

an arteriovenous malformation who earlier had a negative CA. An additional 15 patients (21\%) underwent CA. Of these, 3 revealed a vascular malformation and 2 showed changes suggestive of a vasculitis or vasculopathy. In all instances, a preangiogram MRI/magnetic resonance angiography had correctly identified these findings. Thus, when assessing the diagnostic yield of MRI in this subset of 27 patients who had their ICH cause determined either by pathology and/or conventional CA, MRI had a sensitivity (and negative predictive value) of $93 \%$ (confidence interval 64-99) and a specificity (and positive predictive value) of $100 \%$ (confidence interval $73-$ 100).

Both the stroke and the general neurologist were more likely to reach the reference 'gold standard' ICH diagnosis after reviewing the MRI. The stroke neurologist changed diagnostic category to the correct final diagnosis in $10(14 \%)$ patients and the general neurologist in 13 (19\%) patients after review of the MRI. In 60\% of cases, the change was prompted by features on the MRI remote from the index bleed (e.g. the presence of microhemorrhages in other brain regions). Diagnostic confidence also increased after MRI review both for the stroke neurologist (16 patients, 23\%) and the general neurologist (15 patients, $21 \%$ ). Thus, the overall diagnostic yield of early MRI was 37\% for the stroke neurologist and $40 \%$ for the general neurologist. Two patient examples are shown in figures 1 and 2. In one patient, MRI review changed the diagnostic impression from 'possible brain tumor' to 'hemorrhage secondary to CAA' (fig. 1). In the other pa- tient, MRI review changed the diagnostic impression from 'ICH caused by long-standing hypertension' to 'ICH secondary to a vascular malformation (i.e. cavernous angioma'; fig. 2). In 4 patients (6\%), the general neurologist changed the diagnostic category incorrectly after MRI review. In 3 of these this was caused by interpreting a thin rim of perihematomal restricted diffusion (caused by artifact from blood products) as evidence for hemorrhagic transformation of an ischemic stroke. This did not occur for the stroke neurologist.

The diagnostic yield of MRI did not differ with age, sex, hypertension or the presence of associated subarachnoid hemorrhage or IVH (table 3). Furthermore, the yield was similar for patients with a 'typical' hypertensive ICH (i.e. patients with a history of hypertension who presented with an ICH in the basal ganglia, thalamus or posterior fossa) and those with an 'atypical ICH' (lobar or isolated IVH or multiple hematomas or absence of hypertension; 35 vs. $41 \%$; $\mathrm{p}=\mathrm{NS}$ ). In addition, we did not observe a decrease in diagnostic yield of MRI in patients with a 'typical ICH' and older age (table 3). Thus, even in the subset of patients with a 'classic' hypertensive ICH, acute MRI changed either the initial diagnostic impression or the diagnostic confidence in 35\% of patients.

Eighteen patients had an additional MRI in the chronic phase of the ICH (i.e. beyond 60 days). Three of these had an ICH of 'unknown' etiology after the initial MRI. In 2 of these patients, the final diagnosis was changed after the chronic MRI to CAA and vascular malforma- 

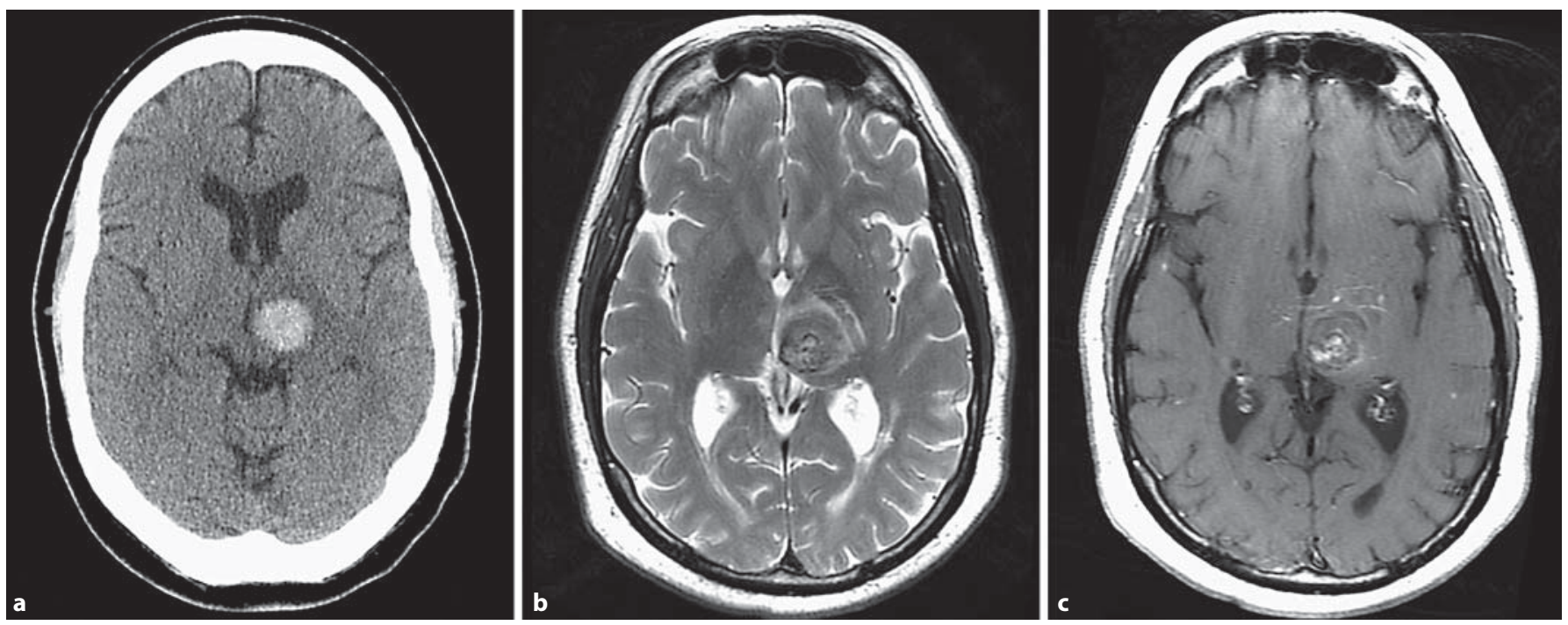

Fig. 2. Brain imaging studies in a 60-year-old hypertensive male with a spontaneous left thalamic ICH. The CT diagnosis of hypertensive ICH (a) was changed after MRI review to ICH caused by a cavernous angioma (b, c). MRI shows a well-circumscribed lesion with a heterogenous reticulated core on the $\mathrm{T}_{2}$-weighted sequence (b) and an associated developmental venous anomaly with a 'caput medusae' appearance on the $\mathrm{T}_{1}$-weighted postcontrast sequence (c). This diagnosis was confirmed surgically.

Table 3. Subgroup analysis of diagnostic yield of MRI in 70 patients with spontaneous ICH

\begin{tabular}{|c|c|c|}
\hline Subgroup & $\begin{array}{l}\text { Diagnosis or confidence } \\
\text { changed by MRI }(n=27)\end{array}$ & $\mathrm{p}$ value \\
\hline Age $\leq 45(n=9)$ vs. $>45$ years $(n=61)$ & $2(22)$ vs. $25(41)$ & 0.47 \\
\hline Males $(n=42)$ vs. females $(n=28)$ & $14(33)$ vs. $13(46)$ & 0.27 \\
\hline History of HTN $(\mathrm{n}=49)$ vs. no HTN $(\mathrm{n}=21)$ & $18(37)$ vs. $9(43)$ & 0.63 \\
\hline HTN + 'typical ICH' $(\mathrm{n}=26)^{1}$ vs. others $(\mathrm{n}=44)$ & $9(35)$ vs. $18(41)$ & 0.60 \\
\hline HTN + 'typical ICH' + age $>45(n=23)$ vs. others $(n=47)$ & 9 (39) vs. 18 (38) & 1.0 \\
\hline HTN + 'typical ICH' + age $>55(n=17)$ vs. others $(n=53)$ & $7(41)$ vs. $20(38)$ & 0.81 \\
\hline HTN + 'typical ICH' + age $>65(n=10)$ vs. others $(n=60)$ & $3(30)$ vs. $24(40)$ & 0.73 \\
\hline Lobar ICH $(\mathrm{n}=23)$ & $8(35)$ & \\
\hline Basal ganglia ICH $(\mathrm{n}=17)$ & $6(35)$ & \\
\hline Thalamus ICH $(n=9)$ & $3(33)$ & \\
\hline Brainstem ICH $(n=2)$ & $2(100)$ & \\
\hline Cerebellar ICH $(n=7)$ & $2(29)$ & \\
\hline Isolated IVH $(\mathrm{n}=5)$ & $2(40)$ & \\
\hline Multiple hematomas $(n=7)$ vs. single hematoma $(n=63)$ & $4(57)$ vs. $23(37)$ & 0.42 \\
\hline ICH with associated SAH $(n=9)$ vs. ICH without associated SAH $(n=61)$ & $2(22)$ vs. $25(41)$ & 0.47 \\
\hline ICH with associated IVH $(n=24)$ vs. ICH without associated IVH $(n=46)$ & $10(42)$ vs. $17(37)$ & 0.70 \\
\hline
\end{tabular}

HTN = Hypertension; SAH = subarachnoid hemorrhage. Figures in parentheses indicate percentages.

${ }^{1}$ Typical location of hypertensive hematoma includes basal ganglia, thalamus, brainstem and cerebellum. 
tion based on interval development of numerous subcortical microbleeds in one and detection of an arteriovenous malformation after hematoma resorption in the other.

The general neurologist and the stroke neurologist reported that the additional information obtained from the MRI would have affected their patient management in 21 and $20 \%$ of patients, respectively. Such changes included decisions about (a) withholding or restarting antiplatelet or anticoagulation therapy, (b) degree of blood pressure control (e.g. tighter blood pressure control in a patient with an untreated vascular malformation), and (c) decisions regarding the need for further diagnostic workup (e.g. in patients for whom MRI review resulted in increased diagnostic confidence, unnecessary additional diagnostic workup could be avoided). The impact of MRI on patient management was particularly high in patients with ICH attributed to hemorrhagic transformation of an ischemic stroke, CAA, vascular malformations or neoplasms (table 2). The relative impact of individual MRI sequences is shown in table 4 . The gradient echo, fluidattenuated inversion recovery and DWI sequences were used most frequently in the decision making to change diagnostic category, diagnostic confidence or management.

\section{Discussion}

Despite the proven superior sensitivity of MRI in the detection of structural brain lesions as compared to CT, it remains unclear whether MRI affects the diagnostic impression or management of ICH patients. Current guidelines on $\mathrm{ICH}$ management do not provide specific recommendations regarding the diagnostic workup of ICH because of the paucity of data $[18,19]$. One retrospective study used predefined criteria to determine whether MRI provided new diagnostic information in ICH [20]. MRI was obtained in $23 \%$ of patients and provided a new diagnosis in $22 \%$ of these. A limitation of that study, however, was that MRIs were obtained only in $23 \%$ of all ICH patients, potentially overestimating its yield. Another retrospective study including 47 patients reported a diagnostic yield of MRI of 36\% [13]. Neither study assessed the potential impact of MRI on patient management.

The results of our study suggest a meaningful clinical benefit of routine brain MRI performed within the first week following symptom onset in patients with a spontaneous ICH or isolated IVH. Based on the MRI findings,
Table 4. Relative impact (\%) of individual MRI sequences on diagnostic category, diagnostic confidence and management in 70 patients with spontaneous ICH (average of 2 observers)

\begin{tabular}{lccc}
\hline MRI sequence & $\begin{array}{l}\text { Change in } \\
\text { diagnostic } \\
\text { category } \\
(\mathrm{n}=11.5)\end{array}$ & $\begin{array}{l}\text { Change in } \\
\text { diagnostic } \\
\text { confidence } \\
(\mathrm{n}=15.5)\end{array}$ & $\begin{array}{l}\text { Change in } \\
\text { management } \\
(\mathrm{n}=14.5)\end{array}$ \\
& & & \\
\hline $\begin{array}{l}\text { Conventional sequences } \\
\left.\mathrm{T}_{1} \text {-weighted ( } \mathrm{n}=70\right)\end{array}$ & 0 & 0 & 0 \\
$\mathrm{~T}_{2}$-weighted $(\mathrm{n}=69)$ & 13 & 6 & 10 \\
$\mathrm{~T}_{1}$-weighted with gadolinium & & & \\
$\quad(\mathrm{n}=44)$ & 13 & 13 & 14 \\
\hline Newer or less routine sequences & & & \\
GRE $(\mathrm{n}=69)$ & 43 & 52 & 48 \\
FLAIR ( $=69)$ & 17 & 39 & 24 \\
MRA ( $\mathrm{n}=61)$ & 4 & 6 & 7 \\
DWI ( $=63)$ & 35 & 19 & 21 \\
MRV ( $\mathrm{n}=6)$ & 17 & 33 & 17
\end{tabular}

GRE = Gradient echo imaging; FLAIR = fluid-attenuated inversion recovery; MRA = magnetic resonance angiography; $\mathrm{MRV}=$ magnetic resonance venography.

diagnostic category or confidence was changed in more than one third of patients $(27 / 70=39 \%)$. Furthermore, the MRI findings were judged to affect management decisions in one fifth of patients. The benefit of MRI appeared similar for the stroke expert and the general neurologist.

The patients in our study were consecutive patients identified from our stroke database over a 5-year time period. During this time 34 of $123 \mathrm{ICH}$ patients did not undergo MRI at our institution. We did not record why these patients did not have MRI. It is possible that this group was different from the study cohort and that the yield of MRI would have been different. However, even if we assumed a 'worst case' scenario (the diagnostic yield of MRI in the 53 patients who were not included in our data set could have been $0 \%$ ), the overall diagnostic yield of MRI in our study would still have been $22 \%$, which we believe is clinically relevant.

The results of our study suggest that the routine use of MRI may be beneficial in all spontaneous ICH patients. The clinical yield of MRI was noted in every etiological category, but was highest in patients with ICH attributed to ischemic stroke, CAA, vascular malformation or neoplasm. This finding is consistent with a previous study showing that CAA and vascular malformations were the 
etiologies most frequently identified by MRI [20]. It should be kept in mind that in ICH due to a neoplasm, obtaining MRI within the first 3 days may help avoid masking of tumor characteristics by methemoglobin that is present in the subacute phase.

We did not observe a substantial differential diagnostic yield of MRI based on patient age, presence of hypertension or hematoma location. Even in the subset of patients with a 'classic' hypertensive ICH, MRI changed either the initial diagnostic impression or confidence in $35 \%$. This finding is different from prior studies looking at the utility of CA in deep ICH $[8,21]$; however, the diagnostic yield of MRI as measured in this study goes beyond identifying an underlying vascular abnormality. MRI can reveal causes for ICH based on findings in the brain parenchyma that cannot be identified on vesselonly imaging studies (e.g. tumors, cerebral infarction and tissue changes consistent with CAA and chronic hypertension) $[13,20]$. Furthermore, with only a limited number of patients in each subgroup, we may not have detected a real difference.

The MRI sequences with the greatest yield were gradient echo, fluid-attenuated inversion recovery and DWI which should be included in all ICH patients who undergo MRI $[13,20]$. More advanced MRI technologies, notably those using parallel imaging, will likely overcome some of the limitations of conventional DWI (low spatial resolution, image distortion near paranasal sinuses and skull base, and magnetic susceptibility artifacts due to blood products), further enhancing the utility of MRI in spontaneous ICH [22-27].

It has been reported that the large majority of spontaneous ICHs are 'primary', i.e. caused either by longstanding hypertension or CAA or both $[28,29]$. Some autopsy series, however, have not confirmed this observation [30]. In this study, only half of the patients had a final diagnosis of ICH due to hypertension and/or CAA. It is possible that our patient population in a tertiary referral center does not reflect the typical distribution of ICH etiologies due to a referral bias. Furthermore, our study population may not be a representative sample of all $\mathrm{ICH}$ patients seen at our institution during the study period since not all spontaneous ICH patients had undergone MRI during that time. Alternatively, it is conceivable that the frequent use of MRI in ICH patients at our institution unmasks secondary hemorrhage causes in patients whose hematomas would have otherwise been attributed to a 'primary' cause. The patient in figure 2 is one such example. Based on the history and location of the $\mathrm{ICH}$ on the CT scan, the ICH was initially attributed to long- standing hypertension until the MRI showed an underlying cavernous angioma. Thus, MRI in this patient affected diagnosis and management. The patient underwent surgical resection of the cavernous angioma to prevent recurrent ICH.

One of the limitations of this study is the validity of the reference standard, which incorporated the results of the MRI. We decided to base our reference standard on the ICH etiology determined by an independent investigator after review of all the available patient information including medical records, CT imaging, MRI, cerebral angiograms, laboratory results, other diagnostic tests, pathology and patient follow-up. Certainly, a gold standard based on brain pathology and/or CA would have been preferable, but this was not feasible in most cases. It is reassuring, however, that in the subset of 27 patients in whom ICH etiology was confirmed by pathology or CA, the sensitivity and specificity of MRI for ICH cause was 93 and $100 \%$, respectively. One other limitation of this study is its retrospective nature and its results should be interpreted within the limits of such a study design. Further, our study design does not allow for the assessment of the practical aspects of obtaining routine MRI in ICH patients or for assessing changes in patient outcome based on MRI-related changes in diagnosis or management. In addition, MRI yield was determined after review of noncontrast CT. Potentially more sensitive CT imaging techniques including contrast-enhanced CT and CT angiography will likely impact the diagnostic yield of MRI in ICH patients [31, 32]. Finally, it is possible that the diagnostic yield of MRI may be different in the chronic phase after resorption of the hematoma. We were unable to address this issue since only a small number of our patients had repeat MRI in the chronic phase. As systematic data on the utility of chronic MRI in spontaneous ICH are lacking, this issue deserves further study.

\section{Acknowledgement}

C.A.C.W. is funded by NIH grants R01 NS034866-08 and R01 HL089116-01A2. 


\section{References}

$\nabla_{1}$ Feigin VL, Lawes CM, Bennett DA, BarkerCollo SL, Parag V: Worldwide stroke incidence and early case fatality reported in 56 population-based studies: a systematic review. Lancet Neurol 2009;8:355-369.

$\checkmark 2$ Brott T, Thalinger K, Hertzberg V: Hypertension as a risk factor for spontaneous intracerebral hemorrhage. Stroke 1986;17:10781083.

-3 Ohtani R, Kazui S, Tomimoto H, Minematsu $\mathrm{K}$, Naritomi H: Clinical and radiographic features of lobar cerebral hemorrhage: hypertensive versus non-hypertensive cases. Intern Med 2003;42:576-580.

4 Barnes B, Cawley CM, Barrow DL: ICH secondary to vascular lesions. Neurosurg Clin North Am 2002;13:289-297.

5 McEvoy AW, Kitchen ND, Thomas DG: Intracerebral haemorrhage and drug abuse in young adults. Br J Neurosurg 2000;14:449454.

6 Yoshida Y, Yoshimoto T, Shirane R, Sakurai Y: Clinical course, surgical management, and long-term outcome of moyamoya patients with rebleeding after an episode of ICH: an extensive follow-up study. Stroke 1999;30:2272-2276.

-7 Broderick JP, Adams HP Jr, Barsan W, Feinberg W, Feldmann E, Grotta J, Kase C, Krieger D, Mayberg M, Tilley B, Zabramski JM, Zuccarello M: Guidelines for the management of spontaneous ICH: a statement for healthcare professionals from a special writing group of the stroke council, American Heart Association. Stroke 1999;30:905-915.

$\checkmark 8$ Zhu XL, Chan MS, Poon WS: Spontaneous intracranial hemorrhage: which patients need diagnostic cerebral angiography? A prospective study of 206 cases and review of the literature. Stroke 1997;28:1406-1409.

$\checkmark 9$ Kidwell CS, Chalela JA, Saver JL, Starkman S, Hill MD, Demchuk AM, Butman JA, Patronas N, Alger JR, Latour LL, Luby ML, Baird AE, Leary MC, Tremwel M, Ovbiagele B, Fredieu A, Suzuki S, Villablanca JP, Davis S, Dunn B, Todd JW, Ezzeddine MA, Haymore J, Lynch JK, Davis L, Warach S: Comparison of MRI and CT for detection of acute ICH. JAMA 2004;292:1823-1830.

-10 Fiebach JB, Schellinger PD, Gass A, Kucinski T, Siebler M, Villringer A, Olkers P, Hirsch JG, Heiland S, Wilde P, Jansen O, Röther J, Hacke W, Sartor K; Kompetenznetzwerk Schlaganfall B5: Stroke magnetic resonance imaging is accurate in hyperacute intracerebral hemorrhage: a multicenter study on the validity of stroke imaging. Stroke 2004;35: 502-506.
11 Oppenheim C, Touzé E, Hernalsteen D, Peeters A, Lamy C, Mas JL, Meder JF, Cosnard G: Comparison of five MR sequences for the detection of acute intracranial hemorrhage. Cerebrovasc Dis 2005;20:388-394.

12 Kidwell CS, Wintermark M: Imaging of intracranial haemorrhage. Lancet Neurol 2008;7:256-267.

13 Young N, Vladica P, Soo YS, Ho D: Acute intracerebral haematomas: assessment for possible underlying cause with MRI scanning Australas Radiol 1993;37:315-320.

14 Renowden SA, Molyneux AJ, Anslow P, Byrne JV: The value of MRI in angiogramnegative intracranial haemorrhage. Neuroradiology 1994;36:422-425.

15 Fazekas F, Kleinert R, Roob G, Kleinert G, Kapeller P, Schmidt R, Hartung HP: Histopathologic analysis of foci of signal loss on gradient-echo $\mathrm{T}_{2}{ }^{*}$-weighted $\mathrm{MR}$ images in patients with spontaneous ICH: evidence of microangiopathy-related microbleeds. AJNR Am J Neuroradiol 1999;20:637-642.

16 Tsushima Y, Aoki J, Endo K: Brain microhemorrhages detected on $\mathrm{T}_{2}{ }^{*}$-weighted gradient-echo MR images. AJNR Am J Neuroradiol 2003;24:88-96.

17 Evans AL, Coley SC, Wilkinson ID, Griffiths PD: First-line investigation of acute intracerebral hemorrhage using dynamic magnetic resonance angiography. Acta Radiol 2005; 46:625-630.

18 Broderick J, Connolly S, Feldmann E, Hanley D, Kase C, Krieger D, Mayberg M, Morgenstern L, Ogilvy CS, Vespa P, Zuccarello M; American Stroke Association Stroke Council; High Blood Pressure Research Council; Quality of Care and Outcomes in Research Interdisciplinary Working Group: Guidelines for the management of spontaneous intracerebral hemorrhage in adults: 2007 update: a guideline from the American Heart Association/American Stroke Association Stroke Council, High Blood Pressure Research Council, and the Quality of Care and Outcomes in Research Interdisciplinary Working Group. Stroke 2007;38:2001-2023.

19 Cordonnier C, Klijn CJ, van Beijnum J, AlShahi Salman R: Radiological investigation of spontaneous intracerebral hemorrhage. systematic review and trinational survey. Stroke 2010;41:685-690.

20 Dylewski DA, Demchuk AM, Morgenstern LB: Utility of magnetic resonance imaging in acute ICH. J Neuroimaging 2000;10:78-83.

21 Park J, Hwang YH, Baik SK, Kim YS, Park SH, Hamm IS: Angiographic examination of spontaneous putaminal hemorrhage. Cerebrovasc Dis 2007;24:434-438.
22 Bammer R, Keeling SL, Augustin M, Pruessmann KP, Wolf R, Stollberger R, Hartung HP, Fazekas F: Improved diffusion-weighted single-shot echo-planar imaging (EPI) in stroke using sensitivity encoding (sense). Magn Reson Med 2001;46:548-554.

$\checkmark 23$ Forbes KP, Pipe JG, Karis JP, Heiserman JE: Improved image quality and detection of acute cerebral infarction with propeller diffusion-weighted MRI. Radiology 2002;225: 551-555.

24 Augustin M, Fazekas A, Bammer R: Fast patient workup in acute stroke using parallel imaging. Topics MRI 2004;15:207-219.

25 Bammer R, Schoenberg S: Current concepts and advances in clinical parallel magnetic resonance imaging. Top Magn Reson Imag 2004;15:129-158.

26 Haacke EM, Xu Y, Cheng YC, Reichenbach JR: Susceptibility weighted imaging (SWI). Magn Reson Med 2004;52:612-618.

27 Wycliffe ND, Choe J, Holshouser B, Oyoyo UE, Haacke EM, Kido DK: Reliability in detection of hemorrhage in acute stroke by a new three-dimensional gradient recalled echo susceptibility-weighted imaging technique compared to computed tomography: a retrospective study. J Magn Reson Imaging 2004;20:372-377.

-28 Mohr JP, Caplan LR, Melski JW, Goldstein RJ, Duncan GW, Kistler JP, Pessin MS, Bleich HL: The Harvard Cooperative Stroke Registry: a prospective registry. Neurology 1978; 28:754-762.

29 Broderick J, Brott T, Tomsick T, Leach A: Lobar hemorrhage in the elderly: the undiminishing importance of hypertension. Stroke 1993;24:49-51.

30 McCormick WF, Rosenfield DB: Massive brain hemorrhage: a review of 144 cases and an examination of their causes. Stroke 1973; 4:946-954.

31 Jayaraman MV, Mayo-Smith WW: Multidetector CT angiography of the intra-cranial circulation: normal anatomy and pathology with angiographic correlation. Clin Radiol 2004;59:690-698.

-32 Delgado Almandoz JE, Schaefer PW, Forero NP, Falla JR, Gonzalez RG, Romero JM: Diagnostic accuracy and yield of multidetector CT angiography in the evaluation of spontaneous intraparenchymal cerebral hemorrhage. AJNR Am J Neuroradiol 2009;30: 1213-1221. 